Access to this work was provided by the University of Maryland, Baltimore County (UMBC)

ScholarWorks@UMBC digital repository on the Maryland Shared Open Access (MD-SOAR) platform.

Please provide feedback

Please support the ScholarWorks@UMBC repository by emailing scholarworks-group@umbc.edu and telling us what having access to this work means to you and why it's important to you. Thank you. 


\title{
Terahertz Spectroscopic Studies and Molecular Modeling of the Far-infrared Properties of Electro-optic Polymers
}

\author{
Megan R. Leahy-Hoppa, Xuemei Zheng, and L. Michael Hayden \\ Department of Physics, University of Maryland, Baltimore County, 1000 Hilltop Circle Baltimore, MD 21250 \\ e-mail: mleahy1@umbc.edu
}

\begin{abstract}
We present far-infrared spectra obtained through terahertz spectroscopy of electro-optic polymers and compare those measurements with molecular modeling simulations of the spectra in the $0-5 \mathrm{THz}$ region.

(C) 2005 Optical Society of America

OCIS codes: (300.6270) Spectroscopy, far infrared; (250.2080) Electro-optic polymers.
\end{abstract}

Non-linear optical (NLO) materials and terahertz (THz) opto-electronics have been studied for over 15 years, however, little is known about the properties of NLO and electro-optic (EO) polymers in the far-infrared (far-IR) region. The focus of this study is to examine the far-IR spectra of EO polymers both experimentally through $\mathrm{THz}$ spectroscopy and computationally through molecular modeling. Through the combination of modeling and experimental studies, we hope to improve the predictive capabilities of the models for future material studies.

Using a THz time-domain spectroscopic method [1], we obtain the far-IR absorption spectra of the sample material by comparing the Fourier transform of the sample and reference time-domain electric fields. We use a 1 $\mathrm{mm} \mathrm{ZnTe}$ emitter and $2 \mathrm{~mm}$ ZnTe sensor with the sample placed at the focus of the THz beam in a four-mirror system (Figure 1). Figure 2 shows the far-IR absorption spectrum of poly(methyl methacrylate) (PMMA), polycarbonate (PC), and an NLO polymer we measured using THz spectroscopy. The NLO polymer is composed of an NLO chromophore guest in an amorphous polycarbonate host polymer matrix. The domain of the experimental spectrum is limited by the pulse width of the optical pump $(\sim 200 \mathrm{fs})$ and the frequency response $(<2.5 \mathrm{THz})$ of the millimeter thick ZnTe emitter and sensor.

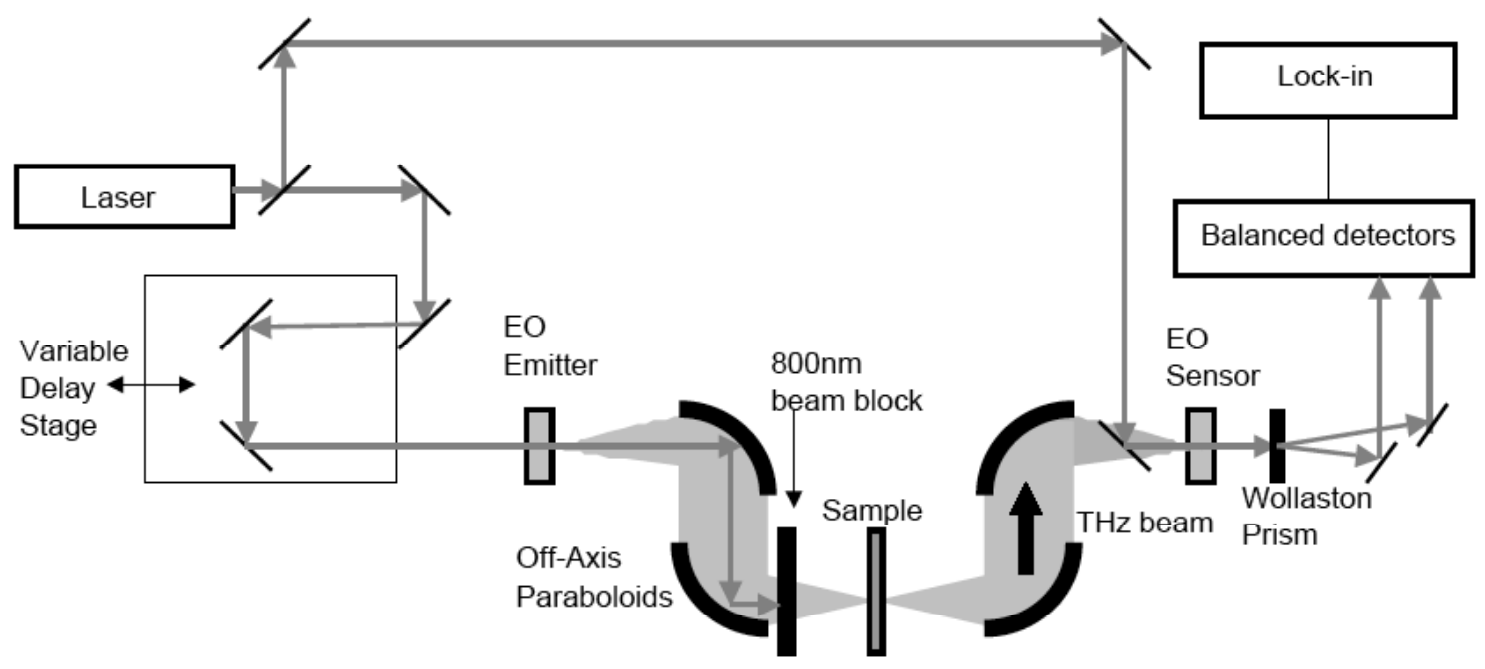

Fig. 1. Experimental set-up for terahertz time-domain spectroscopy 

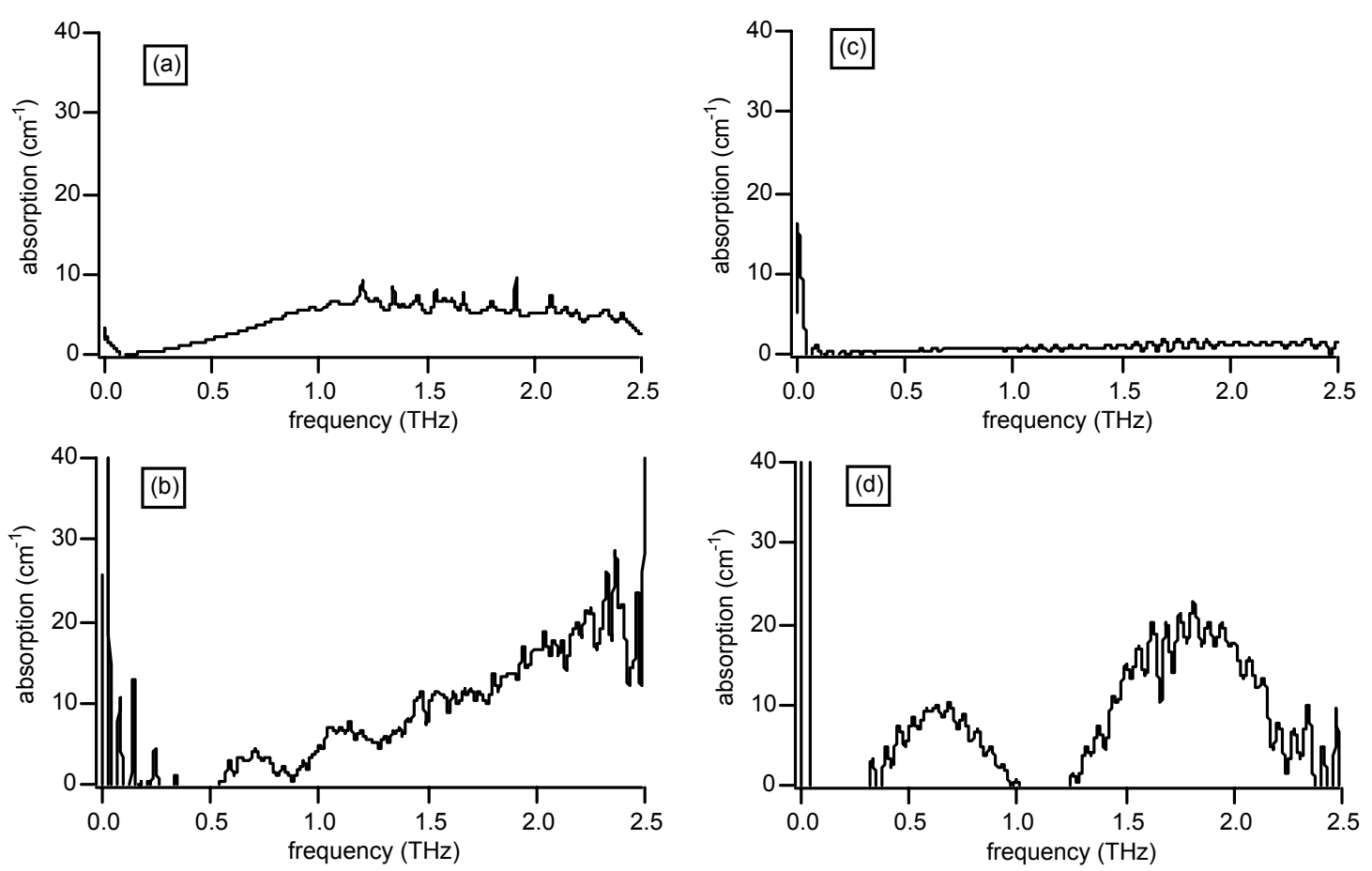

Fig. 2. Far-IR absorption spectrum of PMMA as obtained through terahertz time-domain spectroscopy from (a) a thick PMMA sample, (b) a thin PMMA sample (c) a PC sample, and (d) a PC-based NLO polymer.

Although there are no sharp spectral features in all the materials presented here, in both the thick $(9.4 \mathrm{~mm})$ and thin $(0.214 \mathrm{~mm})$ PMMA samples there is a broad absorption band from $0.5 \mathrm{THz}$ through $2.5 \mathrm{THz}$. The NLO polymer $(0.072 \mathrm{~mm}$ thick) shows two distinct broad absorption bands centered around 0.6 and $1.8 \mathrm{THz}$. The polycarbonate (1.6 mm thick) sample does not seem to absorb in the low $\mathrm{THz}$ region significantly.

We use two different methods for calculating the far-IR absorption spectra from molecular modeling simulations: (1) Normal Coordinate Analysis (NCA) with semi-empirical and density functional (DFT) levels of theory and (2) molecular dynamics (MD). NCA expands the potential energy function of the material in question in a Taylor series. MD employs the Fourier transform of the Dipole Autocorrelation Function (DACF), which utilizes the coordinate trajectories of the atoms to calculate the normal modes of motion of the system.

MD analysis has some advantages over NCA. NCA assumes small amplitudes of vibration about a fixed equilibrium geometry and all vibrations are harmonic. These two assumptions do not necessarily account for low frequency motions of polymeric chains and do not allow for the motions that pass between low energy chains [2] but may be sufficient for the analysis of small individual molecules. In contrast, MD does not assume harmonic motions nor small amplitudes. Moreover, large systems of molecules cannot be studied through quantum or semiempirical method, due to computational limitations, hence MD must be used.

Previous work in modeling the far-IR spectra of polymers has shown agreement between the predicted spectra and experimental data in the main features of the spectra for a few materials [3]. We are extending those methods to include organic EO materials in the far-IR using semi-empirical and DFT techniques with NCA. Figures $3 \mathrm{a}$ and $3 \mathrm{~b}$ show the IR absorption spectra of PMMA as calculated using these two methods. Comparison of the PMMA spectra in Figures $3 \mathrm{a}$ and $3 \mathrm{~b}$ reveals differences between the semi-empirical and DFT techniques using normal coordinate analysis, as well as differences between the experimental spectra (Figure 2a and 2b) and the calculated spectra. The graphs in Figure 3 show the intensity of normal modes of vibration of the polymer chain during the simulation as a function of the frequency of vibration. Both the frequency of normal modes and the intensity associated with each mode differ between the semi-empirical and DFT techniques. Ab-initio modeling techniques such as DFT involve calculations considering all the electrons in the molecules, whereas semi-empirical techniques use empirical data and information from ab-initio calculations to parameterize and make approximations to the calculations. In both these techniques, computational limitations allow only small molecules to be investigated. The differences between the two spectra in Figure 3 arise from the discrepancy between the two methods in the ways the electrons are treated in the calculations. 
MC3
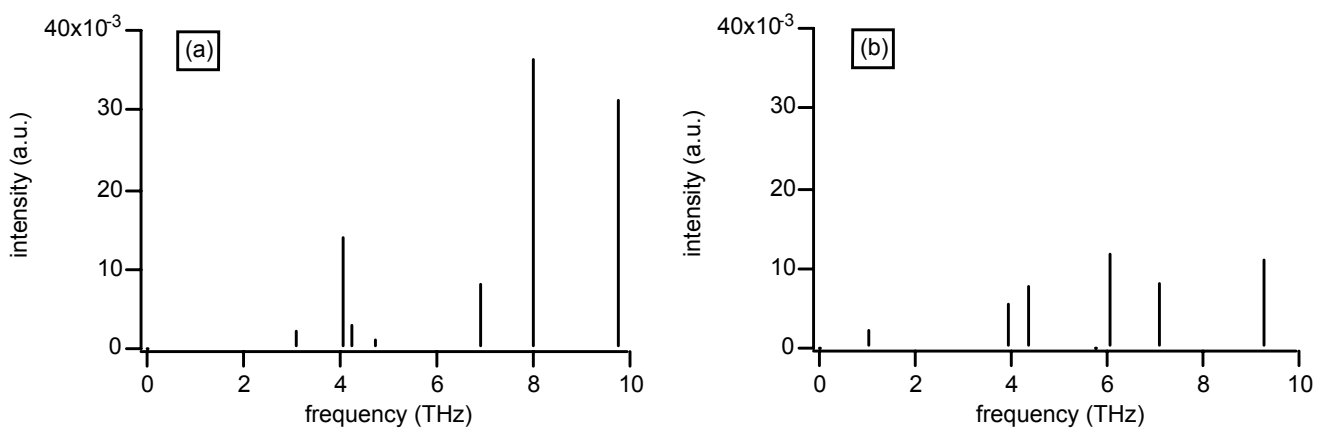

Fig. 3. Far-IR absorption spectra of PMMA as calculated through (a) NCA using semi-empirical theory and (b) NCA using density functional theory.

The spectral information we obtained from the semi-empirical and DFT simulations as seen in Figure 3 do not show absorption peaks in the $0-2.5 \mathrm{THz}$ region of the spectrum. The only spectral information in these two simulations in the region we are currently probing in our $\mathrm{THz}$ spectroscopy experiment is a small peak around 1 $\mathrm{THz}$ in the density functional theory calculation. Based on this information, we would not expect to see much absorption in the experimental THz spectra, however, we do experimentally observe a broad absorption band in the PMMA samples (Figures 1a and 1b). The thick PMMA sample was, in fact, so absorptive that no THZ radiation penetrated through the sample past $1 \mathrm{THz}$. Our experimental results point to the failure of the DFT and semiempirical techniques in this spectral region. Semi-empirical and DFT techniques can only be used on small molecules, which do not necessarily predict the behavior of the bulk material. From Figure 3, we can see that if we increase the bandwidth of our $\mathrm{THz}$ system beyond $5 \mathrm{THz}$ it is possible we will see better agreement with the experimental spectrum.

In order to simulate larger systems and the bulk material, it is necessary to use molecular dynamics. Molecular dynamics uses classical Newtonian mechanics to simulate the time-evolution of the system. Empirical data and abinitio calculations are used to parameterize the calculations so that quantum effects are included even though the calculation is classical. In our previous work, we have studied polystyrene (Figure 4) and found good agreement with literature [2]. Molecular dynamics will be applied in this study to further investigate the spectra of our EO polymers in comparison with our experiment.

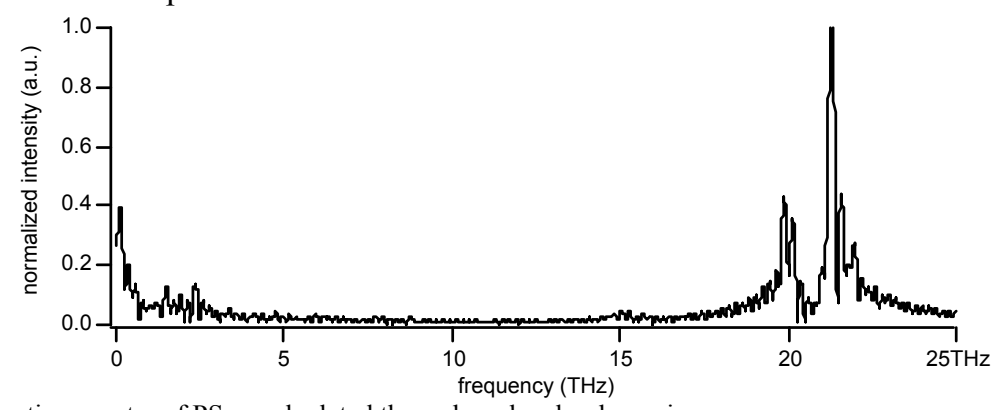

Fig. 4. Far-IR absorption spectra of PS as calculated through molecular dynamics.

Using NLO polymers developed in our lab as emitters and sensors of $\mathrm{THz}$ radiation and a short pulse laser system, we have recently increased the bandwidth of our system from 2.5 to $12 \mathrm{THz}$ without phonon gaps. We are working toward the integration of these polymer emitters and sensors as well as the short pulse laser into our $\mathrm{THz}$ spectroscopy experiment. This increased bandwidth will allow us to extend the spectroscopic information of these and other materials from 2.5 to $12 \mathrm{THz}$. Additionally, we continue to investigate different nonlinear optical polymers through the three methods of molecular modeling mentioned in conjunction with our $\mathrm{THz}$ spectroscopic investigations to improve on the predictive capabilities of our models.

References

[1] M.C. Nuss and J. Orenstein, in Millimeter and sub-millimeter wave spectroscopy of solids, edited by G. Greuner, (Springer, Berlin, 1998), Chap. 2.

[2] A.R. Tiller, Macromolecules, 25, 4605 (1992).

[3] A. Soldera and J-P. Dognon, Macromol. Symp., 119, 157 (1997). 